\title{
Role of diffusion-weighted imaging in differentiation between posterior fossa brain tumors
}

\author{
W. F. Mustafa ${ }^{1}$, M. Abbas $^{1 *}$ (D) and L. Elsorougy ${ }^{2}$
}

\begin{abstract}
Background: Diffusion-weighted imaging (DWI) is an imaging modality using multi-section single-shot spin echo planar imaging (EPI) sequence which is extremely sensitive for detection of water motion within intra, extra, and transcellular regions. This character is important to differentiate between brain tumors either low (benign) or highly (malignant) cellular tumors.

Objective: To evaluate the role of DWI and apparent diffusion coefficient (ADC) in evaluation and differentiation between different brain posterior fossa tumors in children and adults.

Patients and methods: The study included 34 patients with different brain posterior fossa tumors for evaluation by conventional MRI (using 1.5 T MRI PHILIPS Achieva 2.1 Best Netherland) and DWI.

Results: Our study showed that mean ADC values were significantly different between the four groups of posterior fossa tumors in children: juvenile pilocytic astrocytoma (JPA), medulloblastoma, ependymoma, and brain stem glioma while mean ADC values were not significantly different between posterior fossa tumors in the adult group. Regions of interest were manually positioned, and all values were automatically calculated and expressed in $10^{-3} \mathrm{~mm}^{2} / \mathrm{s}$.

Conclusion: DWI is an ideal additional imaging technique, which is a rapid, easy, non-invasive imaging modality, with no contrast injection needed. It has been widely applied in the differentiation between posterior fossa brain tumors and in the diagnosis of various intracranial diseases.
\end{abstract}

Keywords: Diffusion-weighted imaging, Posterior fossa tumors

\section{Introduction}

Brain tumors constitute nearly about 20 to $25 \%$ of all malignancies that occur among children under 15 years of age, and $10 \%$ of brain tumors occur among 15 to 19 year-old children [1].

Brain tumors may be primary (arising from brain itself) or secondary (metastatic from another primary site of cancer). More than 25\% of brain tumors are secondary metastases. Primary brain tumors are the second commonest form of cancers in infants and children under the age of 15 years [2].

Diagnosis of brain tumors by conventional magnetic resonance imaging (MRI) is based on non-contrast T1weighted images (T1WI) and T2-weighted images (T2WI)

\footnotetext{
*Correspondence: dr.m7mdabbas@hotmail.com

${ }^{1}$ Neurology Department, Faculty of Medicine, Mansoura University, Mansoura 35516, Egypt

Full list of author information is available at the end of the article
}

and post-contrast (T1WI). Conventional MRI techniques are considered not enough for grading, classifying, and detecting the aggressiveness of brain tumors [3].

Benign brain tumors such as meningioma and acoustic neuroma form a major part of primary brain tumors [4].

Although conventional MRI provides mainly structural information, such as tumor size, site, and morphological appearance, it does not give a concern about the tumor grade, its aggressiveness, or its histological criteria. For this purpose, studies that are more recent showed that there is a need for other imaging modalities like diffusion-weighted imaging (DWI) [5].

DWI may be helpful to differentiate tumor invasion from normal brain tissue or perifocal brain edema; distinction is very important and will decrease the hazards of biopsy of different brain lesions [6].

In adults, symptoms of brain tumors include persistent headache, vomiting, visual disturbance, hearing or speech 
difficulties, walking problems, personality changes, memory problems, seizures, and loss of weight. While symptoms and signs of posterior fossa tumors in children are the same as those in adults, characteristic headache that worsen at night and early morning and in recumbency that improves with vomiting in addition to behavioral and mental changes, lethargy, irritability, and decreased appetite was also noted. Note that chronic torticollis should be considered as an alarm for the possibility of the presence of a posterior fossa mass.

Our research aimed to evaluate the role of DWI in differentiation between common posterior fossa brain tumors in children and adult patients by calculating the apparent diffusion coefficient (ADC) value of each brain tumor and correlating these results with the histopathological analysis.

\section{Patients and methods}

Thirty-four patients were enrolled in this study. The study was performed at the Neurology and Diagnostic Radiology Department, Mansoura University using a 1.5-T magnetic resonance scanner (Philips Achieva 2.1, Best, Netherlands).

All patients involved in this study were subjected to full medical history, neurological examination, and MRI examination (Conventional and DWI).

Conventional MRI was done in supine position using standard head coil with the head maintained in a neutral position. Sagittal and axial T1-weighted non-contrast images had the following parameters: 600/15 repetition time/echo time (TR/TE), a slice thickness of $5 \mathrm{~mm}$ and a slice gap of $1 \mathrm{~mm}$, a field of view of $230 \times 183 \times 130$, and a matrix of $256 \times 163$.

Axial T2-weighted fast spin echo images had imaging parameters of 4000/100 (TR/TE), a slice thickness of 5 $\mathrm{mm}$ and an interslice gap of $1 \mathrm{~mm}$, a field of view of $230 \times$ $183 \times 130$, and a matrix of $350 \times 224$. Axial fluidattenuated inversion recovery (FLAIR) had imaging parameters of 6000/90 (TR/TE), a slice thickness of $5 \mathrm{~mm}$ and an interslice gap of $1 \mathrm{~mm}$, a field of view of $230 \times 183$ $\times 130$, and matrices of $350 \times 224$ and $256 \times 174$.

Post-contrast-enhanced axial, coronal, and sagittal T1weighted images were obtained. Then, DWI was performed using multi-section single-shot spin echo planar imaging (EPI) sequence with b values 0.5 and $1000 \mathrm{~mm}^{2} / \mathrm{s}$. The diffusion gradients were applied sequentially in the three orthogonal directions. A section of $5 \mathrm{~mm}$ thickness, an interslice gap of $1 \mathrm{~mm}$, a 250 -mm field of view (FOV), and a $128 \times 256$ matrix were used for all images.

The total acquisition time was $80 \mathrm{~s}$. Three types of images were obtained: orthogonal images, trace images, and ADC maps.

Calculation of ADC maps automatically were done by the MRI software. Then, contrast-enhanced TI-weighted images were done after intravenous injection of the contrast agent in a dose of $0.1 \mathrm{~m} \cdot \mathrm{mol} / \mathrm{kg}$. All DW imaging data were transferred to a computer workstation for the determination of the signal intensity and ADC. Each image used for the creation of ADC maps was obtained with specific signal intensity (SI).

Regions of interests (ROIs) were manually positioned, and all values were automatically calculated and expressed in $10^{-3} \mathrm{~mm}^{2} / \mathrm{s}$. ADC measurements were performed using a region of interest (ROI) method, with uniform ellipsoid ROI of $50-100 \mathrm{~mm}^{2}$ area. Three ROIs were placed in each corresponding tumor. The representative value used in the data and statistical analysis was mean value \pm S.D.

The first ROI was placed over a homogenous enhancing region in the central portion of the tumor. Two additional ROIs were placed on the homogenous enhancing areas on different sections; these ROIs were positioned so that an overlapping of the first ROI was avoided. Three ROIs were measured, and their average was calculated.

\section{Statistical methods}

Analysis of data was done using SPSS (statistical program for social science) version 20 . Variables were analyzed as mean values of $\mathrm{ADC} \pm$ standard deviation (SD). Differences among the groups were analyzed using univariate ANOVA. Correlations were determined by using Pearson's test. A $p$ value of $\leq 0.05$ was considered statistically significant, and a $p$ value of $\leq 0.001$ was considered highly significant, while a $p$ value of $>0.05$ was considered non-significant.

\section{Results}

The cohort was composed of 34 patients (19 males and 15 females) with posterior fossa brain tumors. They were divided into two main groups according to their age, pediatric and adult groups, with both groups diagnosed according to radiographic MR findings and histopathological findings except for brain stem glioma in children and metastatic brain tumors in adults. Brain stem glioma was diagnosed only through radiographic findings and its follow-up due to the difficulty of sampling or biopsy. Moreover, this type of brain tumor is inoperable and its treatment just relies on radiotherapy exposure even though metastatic brain tumors are also diagnosed through MRI findings, its follow-up, and the history of patients known to have a primary tumor.

The group of pediatric posterior fossa brain tumors included 11 subjects. Their age was ranged from 2 to 15 years with their mean age being 7.3 years. Four of them were males and seven were females. They were subdivided into three cases of juvenile pilocytic astrocytomas (JPAs), two cases of ependymomas, three cases of medulloblastomas, and three cases of brain stem gliomas. No significant difference was found in comparing between the two methods (1ROI and 3ROIs) in each pediatric subgroup ( $p$ value $=0.572)$. 
All JPA cases had cystic component with a solid mural nodule. The cystic component in all cases appeared with increased SI on DWIs (b0) and with decreased SI on DWIs (b1000) compared to normal appearing brain parenchyma, showing free diffusion the same as that in cerebrospinal fluid (CSF).

Mean ADC values were significantly different between the four groups of posterior fossa tumors in children: JPA, medulloblastoma, ependymoma, and brain stem glioma ( $p$ value $<0.001$ ).

Mean ADC value was significantly different between juvenile pilocytic astrocytoma (JPA) $\left(1.63 \pm 0.05 \times 10^{-3}\right)$ (mean \pm SD) and medulloblastoma $\left(0.73 \pm 0.06 \times 10^{-3}\right)$ (mean $\pm \mathrm{SD}$ ) with a $p$ value of $<0.05$.

Mean ADC value was significantly different between ependymoma $\left(1.15 \pm 0.07 \times 10^{-3}\right)($ mean \pm SD) and medulloblastoma $\left(0.73 \pm 0.06 \times 10^{-3}\right)$ (mean $\left.\pm \mathrm{SD}\right)$ with a $p$ value $<0.05$.

Mean ADC value was significantly different between medulloblastoma $\left(0.73 \pm 0.06 \times 10^{-3}\right)($ mean $\pm \mathrm{SD})$ and brain stem glioma $\left(1.37 \pm 0.12 \times 10^{-3}\right)$ (mean $\left.\pm \mathrm{SD}\right)$ with a $p$ value $<$ of 0.05 .

The group of adult posterior fossa brain tumors included 23 subjects. Their age ranged from 24 to 65 years with their mean age being 46.4 years. Their sex distribution was 15 males and 8 females. Distribution of age decades in this group was between 2 and 15 years. Posterior fossa tumors encountered in the adult group of our study were metastasis in five cases (group 1), cerebellar (infratentorial) meningiomas in four cases (group 2), hemangioblastomas in three cases (group 3), cerebellopontine angle (CPA) acoustic schwannomas in five cases (group 4), CPA epidermoid tumors (cysts) in two cases (group 5), arachnoid cysts in two cases (group 6), and CPA meningiomas in two cases (group 7).

No significant difference was found in comparing between the two methods (1ROI and 3ROIs) of each adult subgroup as shown in Fig. 1 ( $p$ value $=0.641$ ).

All hemangioblastoma cases had cystic component with a solid mural nodule. The cystic component in all cases appeared with increased SI on DWIs (b0) and with decreased SI on DWIs (b1000), in which abnormal MRI sequences were found in DWI abnormal cystic lesions, compared to normal appearing brain parenchyma, showing free diffusion the same as that in CSF.

As a whole, mean ADC value was not significantly different between posterior fossa tumors in adult group

Mean ADC value was considered significant different between epidermoid tumors (cysts) $\left(0.85 \pm 0.07 \times 10^{-3}\right)$ (mean $\pm \mathrm{SD})$ and arachnoid cysts $\left(2.95 \pm 0.02 \times 10^{-3}\right)$ (mean $\pm \mathrm{SD}$ ) with a $p$ value $<0.05$.

\section{Discussion}

The main role of conventional MRI in brain tumors is to distinguish its location, extent, morphology, and relation to important structures, but alone it is deficient in diagnostic examination. Thus, there is a need for additional imaging modalities such as DWI which may aid in diagnosis and differentiation of brain tumors [6].

DWI can give important information about each tumor cellularity and water motion as well as the water content of their related matrix. Also, it can classify brain tumors into low, moderate, and high cellularity, so it is helpful to differentiate, characterize, and distinguish between brain tumors [7].

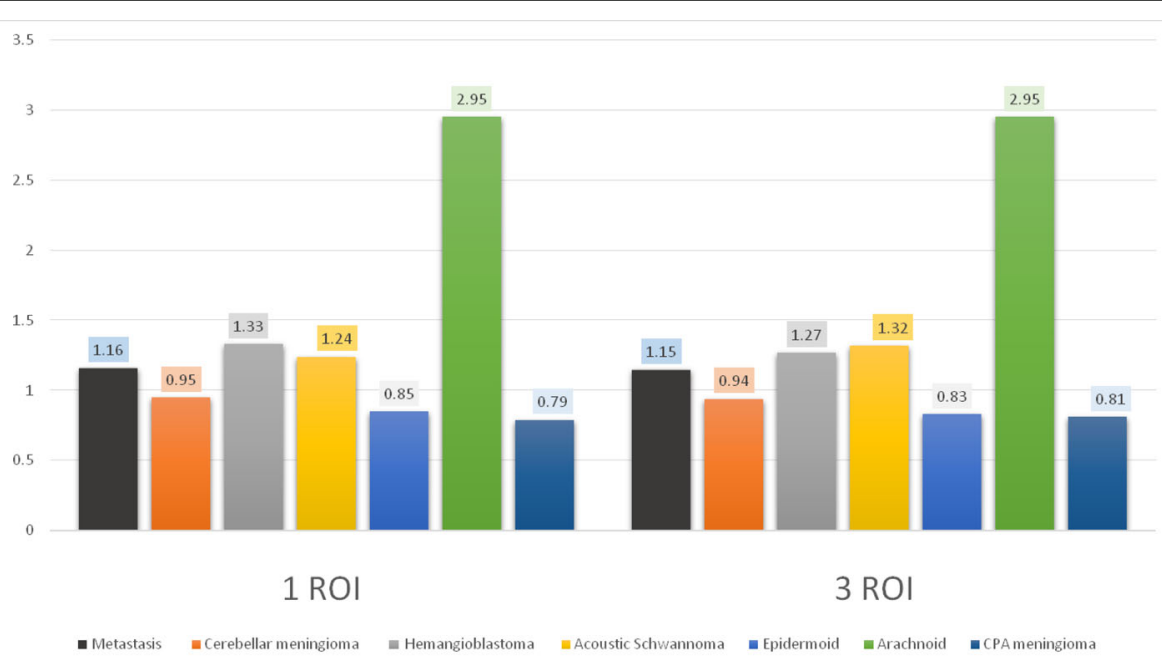

ROI : region of interest

ADC: apparent diffusion coefficient

Fig. 11 ROI versus 3ROls (mean ADC value) of each adult subgroup 

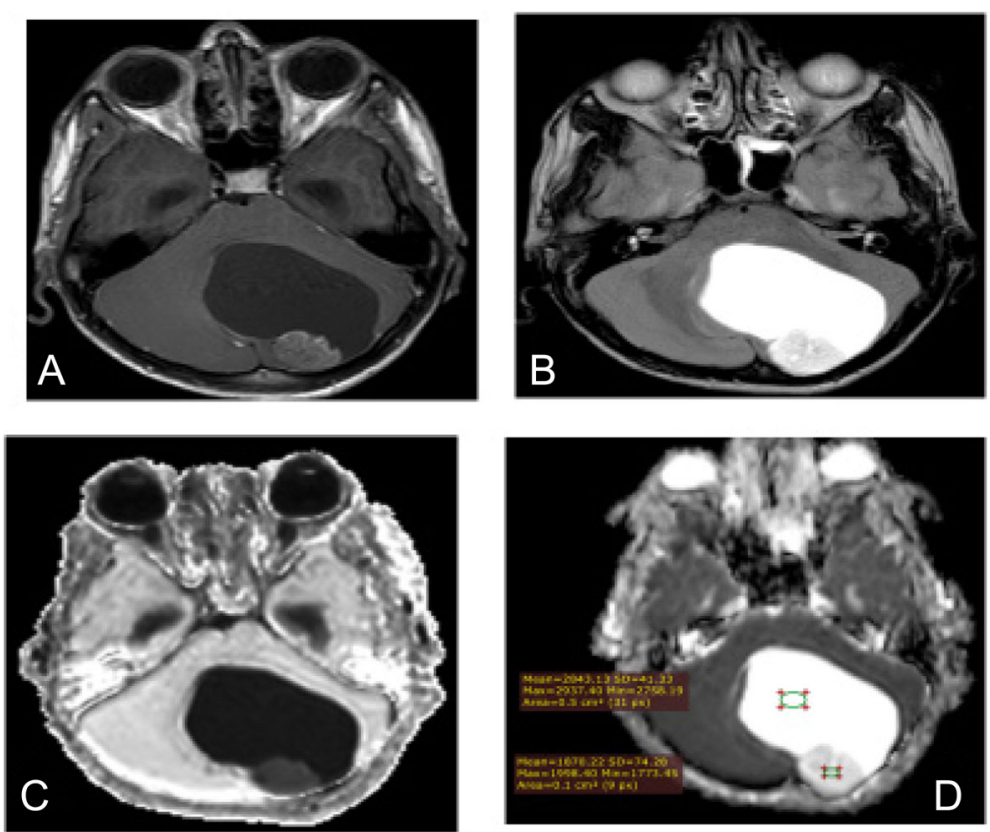

Fig. 2 Juvenile pilocytic astrocytoma in a 9-year-old boy who presented with repeated headache and vomiting. a T1-weighted (T1WI) shows intra-axial cystic space-occupying lesion (SOL) in the left cerebellar hemisphere. b T2-weighted (T2WI) showing hyperintense cystic posterior fossa mass with isointense solid mural nodule attached to the inner side of the cystic mass. Note the cystic components and relatively solid components. c Post-contrast T1WI axial image showing mildly enhancing solid components and faint enhanced cystic wall. d Apparent diffusion coefficient (ADC) map: the cystic component is bright while the solid component is relatively lower than the cystic component with slight diffusion restriction

DWI can assess diffusion of water particles within intra-, extra-, and transcellular spaces by the aid of ADC measurement. In addition to the role of DWI in differentiating most posterior fossa brain tumors, it also participates in preoperative management and in postoperative follow-up for detection of any tumor residual [8].

In this study, we prospectively evaluated the role of ADC values in characterization and differentiation between posterior fossa brain tumors in pediatric and adult patients. Results were correlated to postoperative pathological analysis except for brain stem glioma cases and metastatic brain tumors.

Our study included 34 patients distributed into two main groups: pediatric and adult group according to the age of our patients.

Firstly, the pediatric group included 11 cases and showed a significant difference of mean ADC values between different groups of posterior fossa brain tumors in children: JPA, medulloblastoma, ependymoma, and brain stem glioma.

According to our study results, ADC values were significantly different between JPA (solid nodule) and medulloblastoma.

In addition, we found a significant difference between the ADC values of medulloblastoma and ependymoma. There was no significant difference between ADC value of JPA and $\mathrm{ADC}$ value of ependymoma.
This agree with the findings in Rumboldt and colleagues' study, as their study included 31 children with posterior fossa tumors with their mean age being 10 years and they found a significant difference between the ADC value of JPA and the ADC value of medulloblastoma and also, between the ADC value of ependymoma and the ADC value of medulloblastoma [9].

Ependymomas are well-circumscribed, moderately cellular tumors. The characteristic appearance shows perivascular pseudorosettes (cleared areas and radially arranged cells around blood vessels). As ependymomas progress in malignancy, they also increase in cellularity. In addition, anaplastic ependymomas are also less common than the more benign variants of ependymoma [10].

While using DWI in radiological examination of ependymoma, sometimes there may be an overlap between the types of ependymoma such as classic or anaplastic type also, it is difficult for pathologists to differentiate between them. High ADC value in JPAs and low ADC value in medulloblastomas are mostly secondary to the low cellularity and relatively small nuclear area seen in former tumor types in contrast to the crowded densely packed cells with large nuclei that characterize the latter [11].

Slight diffusion restriction observed in the solid nodule of JPA (Fig. 2) is mostly because of the low tumor cellularity appearance that was confirmed on histopathological 

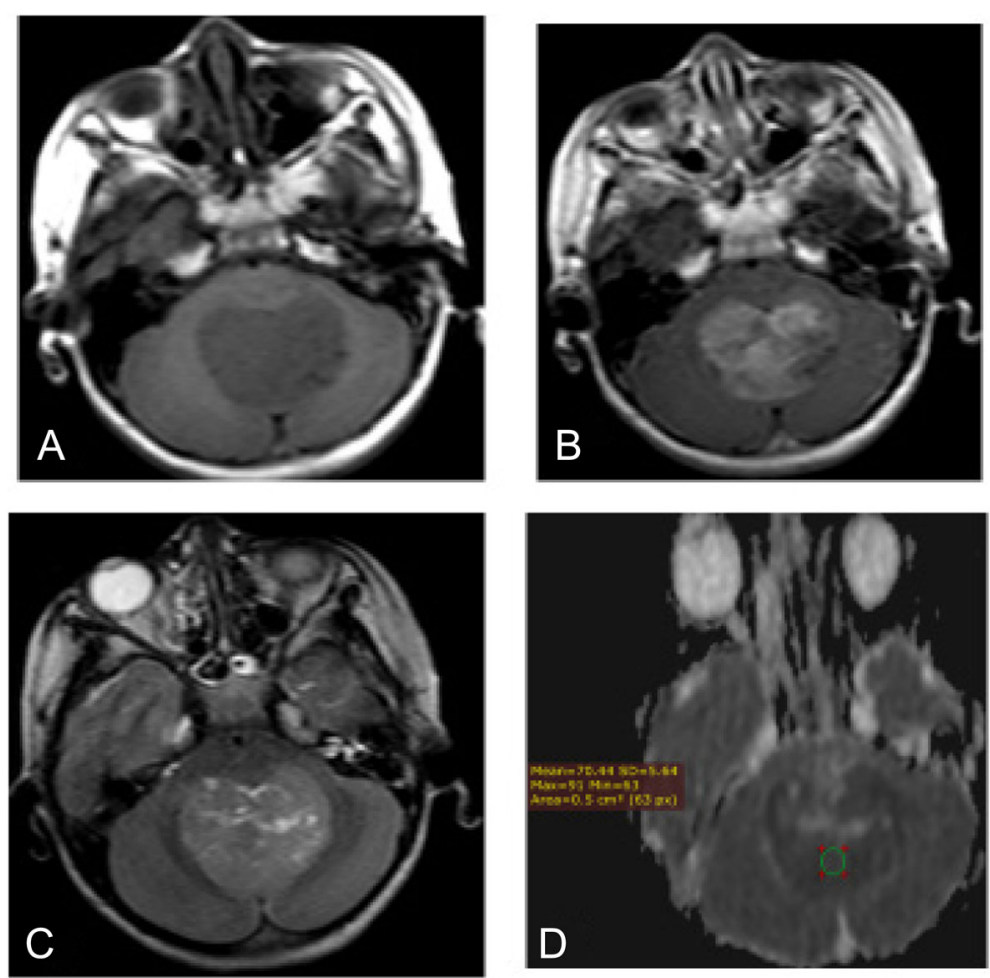

Fig. 3 Typical MRI features of a medulloblastoma in a 3-year-old child who presented with chronic headache 5 months ago. a T1WI shows midline vermian hypointense mass in the posterior fossa causing anterior displacement of the fourth ventricle. $\mathbf{b}$ T2Wl: the mass appears isointense to slightly hyperintense. Few T1WI hypointense and T2WI hyperintense foci suggestive of cystic components. c Post-contrast T1WI axial image showing intense enhancement of the mass. $\mathbf{d}$ Low signal on apparent diffusion coefficient images (ADC), features suggestive of restricted diffusion corresponding to hypercellularity of the mass

analysis. In addition, JPAs have a classic "biphasic pattern, " with alternating light (vacuolated) and denser areas. In JPAs, there are frequently micro-cysts in addition to the macro- cysts seen on imaging studies. The "piloid" cells are characteristic for pilocytic astrocytoma and may be responsible for increased ADC value of JPA (solid nodule) [12].

The high cellularity of medulloblastoma (Fig. 3) is a well-known histologic feature of these tumors. Medulloblastoma characteristically shows no pattern over crowded sheets of small cells with small areas of necrosis which reflects low or decreased ADC values of medulloblastoma [11].

As there is a conflict between ependymoma and medulloblastoma, our results showed no overlap between them, which is in agreement with the study performed by Yamasaki and colleagues who concluded that ADC values were retrospectively $100 \%$ accurate in the differentiation between ependymomas and PNETs [13].

Our results showed no overlap between the mean ADC value of JPA and medulloblastoma. This finding is in contrast to Schneider and colleagues' study, who found overlap between mean ADC value of JPA and medulloblastoma in some cases [14].
Concerning the group of brain stem glioma, we had three cases that were diagnosed radiologically on conventional MRI and its follow-up. They were of diffuse intrinsic type. Brain stem glioma cases were hypointense on T1WI and hyperintense on T2WI with minimal (non-significant) enhancement after contrast administration.

Secondly, the adult group included 23 patients. In our results, we had 4 cases of typical cerebellar "infratentorial" meningiomas (Fig. 4) that were isointense on DWIs obtained at b0 and b1000 which are in harmony with a study achieved by Tantawy and colleagues, who explained 24 cases of typical cerebellar meningiomas appeared as isointense or slightly hyper intense on DWIs obtained at b0 and b1000. Our ADC range value concerning cerebellar meningioma is 0.88 to $1.42 \mathrm{~mm}^{2} / \mathrm{s}$ which is within their range of ADC values from 0.72 to $1.8 \times 10^{-3} \mathrm{~mm}^{2} / \mathrm{s}$ [15].

Regarding acoustic schwannoma tumor, in our study, we had five male patients with tumors located at CPA and extend into the IAC. The ADC value of CPA acoustic schwannoma is higher in relation to the ADC value of CPA meningioma with no significant difference between them. This result is in agreement with a study achieved by Pavlisa and colleagues who found that mean 

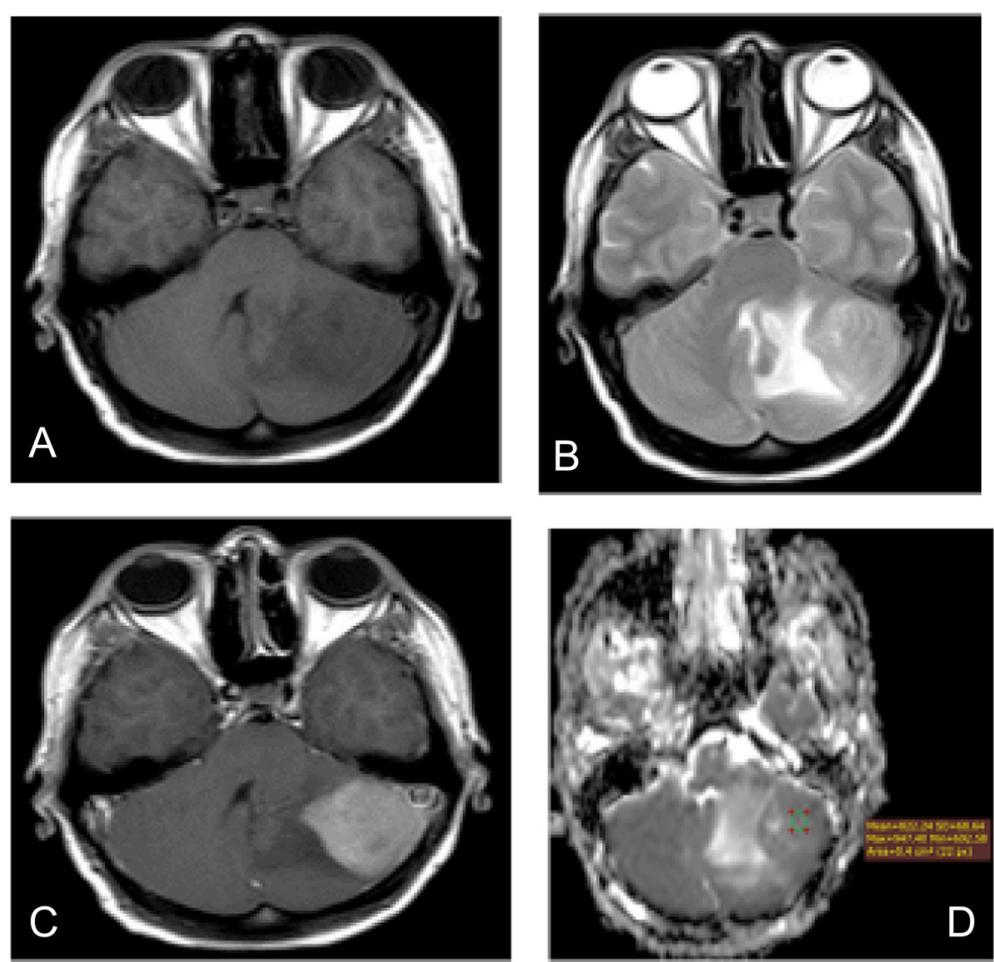

Fig. 4 Typical cerebellar (infratentorial) meningioma in female patient aged 44 years old presented with chronic headache and dizziness 4 months ago. An extra-axial space-occupying lesion (SOL) is seen in the left cerebellar hemisphere lying below and contacting the tentorium cerebelli with moderate perifocal edema, effacement of related cerebellar folia, compression of the fourth ventricle, and minimal rightward shift of midline structures. This mass displays relatively iso to low signal intensity (SI) on T1WI (a), iso SI on T2 WI (b). It shows homogeneous enhancement on post-contrast T1WI (c). It has iso Sl-like brain parenchyma on apparent diffusion coefficient images (ADC) map (d)

ADC value of CPA acoustic schwannoma was higher in relation to mean ADC value of CPA meningioma [16].

Relative higher ADC value of acoustic schwannoma is probably secondary to elongated Schwann cells that may contribute to a higher degree of water content and much more increase of extracellular matrix that mainly affect diffusion properties of acoustic schwannoma [17].

As reported by Lalwani and colleagues, CPA meningiomas rarely expand the internal auditory canal and usually have a broad base against adjacent bone and usually have strong homogeneous enhancement that are not found in CPA schwannoma. On the other hand, acoustic schwannoma expand the IAC with a component inside it, and heterogeneous enhancement is observed after contrast administration [18].

In our study, we had no significant difference between metastatic brain tumors and hemangioblastoma (solid mural nodule) tumors, as regards the ADC values of metastatic brain tumors $(n=5)$ and those of hemangioblastoma $(n=3)$. This finding coincides with a study achieved by Cha study, who found no significant difference between them [19]. In addition, our study results are in agreement with a study achieved by Quadery and colleagues, who reported that mean ADC value of hemangioblastoma was higher in relation to other cerebellar tumors including brain metastasis, medulloblastoma, and ependymoma [20].

Concerning tumor-like cystic lesions, our study had two cases of CPA epidermoid tumors (Fig. 5) and two cases of arachnoid cysts, the former tumors were of low SI on T1WI, with increased SI on T2WI, partially suppressed on FLAIR, with no perifocal edema and minimal contrast enhancement after contrast administration. Also, they were hyperintense on DWI with decreased $\mathrm{ADC}$ values on $\mathrm{ADC}$ mapping denoting restricted diffusion. While the latter cysts were hypointense on T1WI and hyperintense on T2WI also, on DWI, they were hypointense and had high ADC values on ADC map (the same SI as those of CSF) denoting free diffusion. This is in agreement with a study achieved by Lai and colleagues, who studied 11 extra-axial cystic lesions, 9 cases of them were arachnoid cysts that appear hypointense on DWI and hyperintense on ADC mapping [21].

Also, 2 studies included 23 cases of intracranial cysts, 21 of them were epidermoid. They found a significant difference between arachnoid cysts and epidermoid cysts also, and they found that the mean ADC value of epidermoid tumor was relatively 

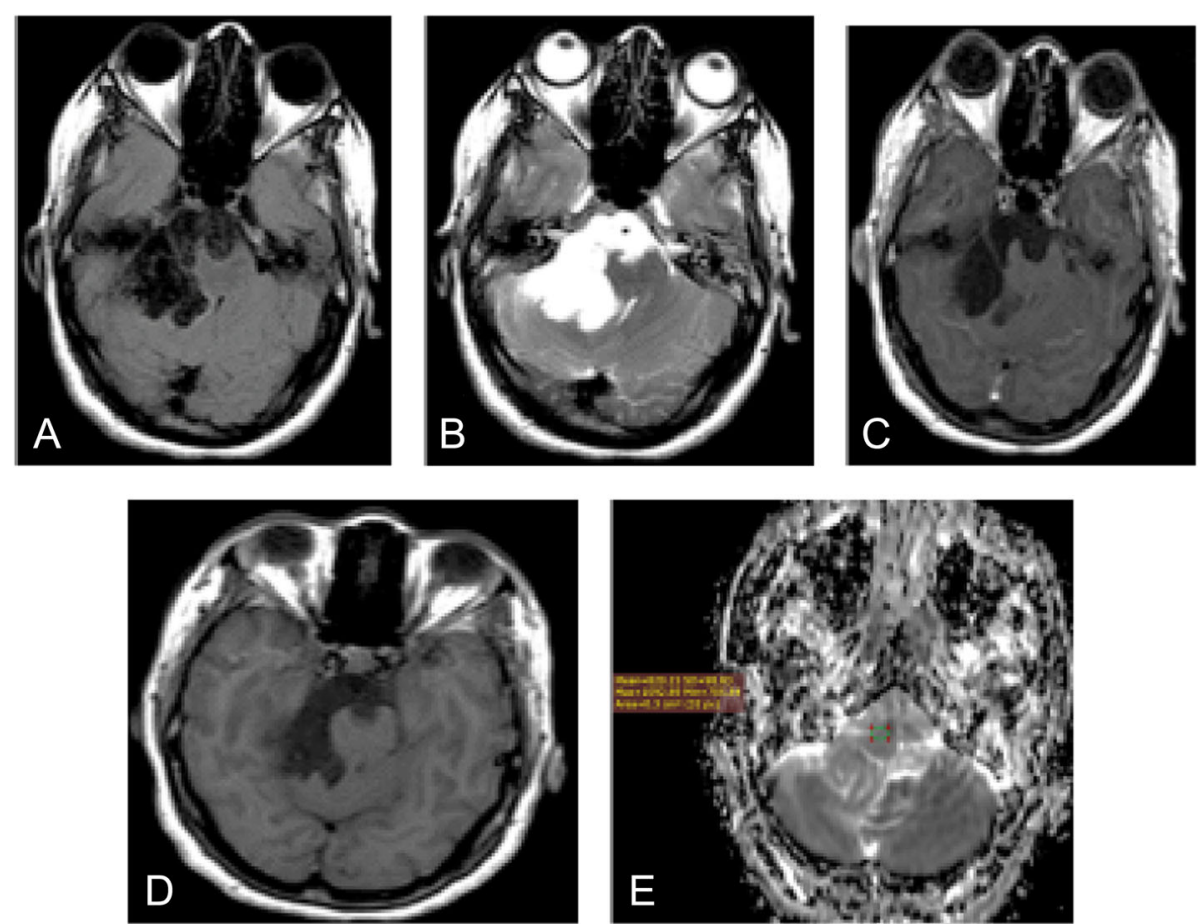

Fig. 5 Epidermoid cyst in 38-year-old male complaining from auditory problems. A lobulated extra-axial cystic lesion is seen in the right cerebellopontine angle (CPA) insinuating itself, compressing the pons, extending into prepontine cistern, and encasing the basilar artery. It displays low T1Wl signal intensity (a) and high T2WI signal intensity cerebrospinal fluid (CSF) like (b). On FLAIR, it shows low SI (partially suppressed SI (c). It shows faint marginal enhancement on post-contrast study (d). It shows iso SI-like brain parenchyma (restricted diffusion); it does not have high CSF SI-like arachnoid cyst (e)

higher than that of our results. In addition, they concluded that the lowest ADC value of all intracranial cysts were epidermoid cysts [22, 23].

The best explanation of low ADC value and high DWI of epidermoid tumor (restricted diffusion) is that the presence of several factors concerning epidermoid tumors like high protein content and high cholesterol content and their increased content of keratinaceous debris [24].

Depending on DWI, a single diagnostic imaging tool, is not enough, as isolated analysis of diffusion properties that concern each tumor cellularity does not usually provide reliable information about differentiation between brain tumors because diagnosis is never based on a single imaging sequence [12].

Also, depending on T1WI and T2WI characteristics of conventional MRI alone is not enough for differentiation between most of posterior fossa brain tumors like JPA, ependymoma, and medulloblastoma [22].

We used another additive imaging modality such as DWI in addition to conventional MRI, and by measuring ADC values of brain tumors mentioned above, we found that ADC values are variable and range from low to high according to the histological cellularity nature of each brain tumor. This can be used in comparing brain tumors and differentiating them into being either benign or malignant tumors.

Limitations of our study included a relatively smaller number of patients in different pediatric and adult groups and having few patients presented with a difficulty in sampling or having biopsy from their lesion.

\section{Conclusion}

DWI is an ideal additional imaging technique which is a rapid, easy, and non-invasive imaging modality, and no contrast injection is needed. It has been widely applied in the differentiation between posterior fossa brain tumors and in the diagnosis of various intracranial diseases.

\section{Abbreviations}

ADC: Apparent diffusion coefficientDWIDiffusion-weighted imagingMRIMagnetic resonance imagingJPAJuvenile pilocytic astrocytomaROIRegion of interestTR/TERepetition time/echo timeEPIEcho planar imagingFOVField of viewSISignal intensity

\section{Acknowledgements}

The authors acknowledge the subjects for their participation and cooperation in this study.

\section{Informed consent}

Informed written consent was obtained from all individual participants included in the study.

We agree to publish in the EJNPN, and the research is only applied here. 


\section{Authors' contributions}

WF and MA contributed in collecting the data and diagnosing cases and writing the manuscript and LE was involved in the MRI studies and diagnosing radiological images. All authors read and approved the final manuscript.

\section{Funding}

This research was supported by the Department of Neurology, Faculty of Medicine at Mansoura University.

\section{Availability of data and materials}

The datasets generated and/or analyzed during the current study are not publicly available due to current Mansoura University regulations and Egyptian legislation but are available from the corresponding author on reasonable request and after institutional approval.

\section{Ethics approval and consent to participate}

All procedures performed in studies involving human participants were in accordance with the ethical standards of the institutional research committee (MFM-IRB) by the date of 3/8/2016.

\section{Consent for publication}

Not applicable

\section{Competing interests}

The authors declare that they have no competing interests.

\section{Author details}

${ }^{1}$ Neurology Department, Faculty of Medicine, Mansoura University, Mansoura 35516, Egypt. ${ }^{2}$ Diagnostic Radiology Department, Faculty of Medicine, Mansoura University, Mansoura 35516, Egypt.

Received: 22 August 2019 Accepted: 27 December 2019

Published online: 06 April 2020

\section{References}

1. Panigrahy A, Blüml S. Neuroimaging of pediatric brain tumors: from basic to advanced magnetic resonance imaging (MRI). J Child Neurol. 2009;24(11):1343-65.

2. Nejat F, El Khashab M, Rutka JT. Initial management of childhood brain tumors: neurosurgical considerations. J Child Neurol. 2008;23(10):1136-48.

3. Nardone V, Tini P, Biondi M, Sebaste L, Vanzi E, De Otto G, et al. Prognostic value of MR imaging texture analysis in brain non-small cell lung cancer oligo-metastases undergoing stereotactic irradiation. Cureus. 2016;8(4).

4. Gurney JG, Kadan-Lottick N. Brain and other central nervous system tumors: rates, trends, and epidemiology. Curr Opin Oncol. 2001;13(3):160-6.

5. Peet AC, Arvanitis TN, Leach MO, Waldman AD. Functional imaging in adult and paediatric brain tumours. Nat Rev Clin Oncol. 2012;9(12):700.

6. Stadnik TW, Demaerel P, Luypaert RR, Chaskis C, Van Rompaey KL, Michotte A, et al. Imaging tutorial: differential diagnosis of bright lesions on diffusionweighted MR images. Radiographics. 2003;23(1):e7-e.

7. Moritani T, Ekholm S, Westesson P-LA. Diffusion-weighted MR imaging of the brain: Springer Science and Business Media; 2009.

8. Yousef AF, Elkharbotly A, Settin M, Mousa Y. Role of diffusion-weighted MR imaging in discrimination between the intracranial cystic masses. Egypt J Radiol Nucl Med. 2014;45(3):869-75.

9. Rumboldt Z, Camacho D, Lake D, Welsh C, Castillo M. Apparent diffusion coefficients for differentiation of cerebellar tumors in children. Am J Neuroradiol. 2006:27(6):1362-9.

10. Kleihues P, Louis DN, Scheithauer BW, Rorke LB, Reifenberger G, Burger PC, et al. The WHO classification of tumors of the nervous system. J Neuropathol Exp Neurol. 2002;61(3):215-25.

11. Ellison D. Classifying the medulloblastoma: insights from morphology and molecular genetics. Neuropathol Appl Neurobiol. 2002;28(4):257-82.

12. Perry A. Pathology of low-grade gliomas: an update of emerging concepts. Neuro Oncol. 2003;5(3):168-78.

13. Yamasaki F, Kurisu K, Satoh K, Arita K, Sugiyama K, Ohtaki M, et al. Apparent diffusion coefficient of human brain tumors at MR imaging. Radiology. 2005; 235(3):985-91.

14. Schneider J, Confort-Gouny S, Viola A, Le Fur Y, Viout P, Bennathan M, et al. Multiparametric differentiation of posterior fossa tumors in children using diffusion weighted imaging and short echo time $1 \mathrm{H}$ MR spectroscopy. J Magn Reson Imaging. 2007;26(6):1390-8.
15. Tantawy HI, Mohamad FF, Ismael AM. The role of advanced MRI techniques in differentiating typical, from atypical and malignant meningiomas. Egypt J Radiol Nucl Med. 2010;41(3):411-9.

16. Pavlisa G, Rados M, Pazanin L, Padovan RS, Ozretic D, Pavlisa G. Characteristics of typical and atypical meningiomas on ADC maps with respect to schwannomas. Clin Imaging. 2008;32(1):22-7.

17. Sadeghi N, Camby I, Goldman S, Gabius H-J, Balériaux D, Salmon I, et al. Effect of hydrophilic components of the extracellular matrix on quantifiable diffusion-weighted imaging of human gliomas: preliminary results of correlating apparent diffusion coefficient values and hyaluronan expression level. Am J Roentgenol. 2003;181(1):235-41.

18. Lalwani AK, Jackler RK. Preoperative differentiation between meningioma of the cerebellopontine angle and acoustic neuroma using MRI. Otolaryngol Head Neck Surg. 1993;109(1):88-95.

19. Cha J, Kim ST, Nam D-H, Kong D, Kim H-J, Kim Y, et al. Differentiation of hemangioblastoma from metastatic brain tumor using dynamic contrastenhanced MR imaging. Clin Neuroradiol. 2017;27(3):329-34.

20. Quadery FA, Okamoto K. Diffusion-weighted MRI of haemangioblastomas and other cerebellar tumours. Neuroradiology. 2003;45(4):212-9.

21. Lai P-H, Hsu S-S, Ding S-W, Ko C-W, Fu J-H, Weng M-J, et al. Proton magnetic resonance spectroscopy and diffusion-weighted imaging in intracranial cystic mass lesions. Surg Neurol. 2007;68:S25-36.

22. Chen S, Ikawa F, Kurisu K, Arita K, Takaba J, Kanou Y. Quantitative MR evaluation of intracranial epidermoid tumors by fast fluid-attenuated inversion recovery imaging and echo-planar diffusion-weighted imaging. Am J Neuroradiol. 2001;22(6):1089-96.

23. Hakyemez B, Aksoy U, Yildiz H, Ergin N. Intracranial epidermoid cysts: diffusion-weighted, FLAIR and conventional MR findings. Eur J Radiol. 2005; 54(2):214-20.

24. Sirin S, Gonul E, Kahraman S, Timurkaynak E. Imaging of posterior fossa epidermoid tumors. Clin Neurol Neurosurg. 2005;107(6):461-7.

\section{Publisher's Note}

Springer Nature remains neutral with regard to jurisdictional claims in published maps and institutional affiliations.

\section{Submit your manuscript to a SpringerOpen ${ }^{\circ}$ journal and benefit from:}

- Convenient online submission

- Rigorous peer review

- Open access: articles freely available online

High visibility within the field

- Retaining the copyright to your article

Submit your next manuscript at $\boldsymbol{\sim}$ springeropen.com 